\title{
Daily Variability in Working Memory is Coupled With Negative Affect: The Role of Attention and Motivation
}

\author{
Annette Brose \\ Max Planck Institute for Human Development, Berlin, Germany
}

\author{
Florian Schmiedek \\ Max Planck Institute for Human Development, Berlin, Germany \\ and German Institute for International Educational Research \\ (DIPF), Frankfurt am Main, Germany \\ Ulman Lindenberger
}

Martin Lövdén

Max Planck Institute for Human Development, Berlin, Germany, Lund University, and Aging Research Center, Karolinska

Institute, Sweden

\begin{abstract}
Across days, individuals experience varying levels of negative affect, control of attention, and motivation. We investigated whether this intraindividual variability was coupled with daily fluctuations in working memory (WM) performance. In 100 days, 101 younger individuals worked on a spatial N-back task and rated negative affect, control of attention, and motivation. Results showed that individuals differed in how reliably WM performance fluctuated across days, and that subjective experiences were primarily linked to performance accuracy. WM performance was lower on days with higher levels of negative affect, reduced control of attention, and reduced task-related motivation. Thus, variables that were found to predict WM in betweensubjects designs showed important relationships to WM at the within-person level. In addition, there was shared predictive variance among predictors of WM. Days with increased negative affect and reduced performance were also days with reduced control of attention and reduced motivation to work on tasks. These findings are in line with proposed mechanisms linking negative affect and cognitive performance.
\end{abstract}

Keywords: intraindividual variability, working memory, affect, motivation, attention

Accounts of within-person variation of psychological phenomena are manifold. For example, patterns of person-context interactions vary depending on situational characteristics as well as on evaluations of situations (Mischel \& Shoda, 1995); affect varies, reflecting for example diurnal rhythms or a stressful encounter (Bolger, DeLongis, Kessler, \& Schilling, 1989). Whereas short-

This article was published Online First July 25, 2011.

Annette Brose and Ulman Lindenberger, Center for Lifespan Psychology, Max Planck Institute for Human Development, Berlin, Germany; Florian Schmiedek, Center for Lifespan Psychology, Max Planck Institute for Human Development, Berlin, Germany and German Institute for International Educational Research (DIPF) Frankfurt am Main, Germany; Martin Lövdén, Center for Lifespan Psychology, Max Planck Institute for Human Development, Berlin, Germany, Department of Psychology, Lund University, Lund, Sweden and Aging Research Center, Karolinska Institute, Sweden.

The COGITO Study was supported by the Max Planck Society, including a grant from the innovation fund of the Max Planck Society (M.FE.A.BILD0005), and the Sofja Kovalevskaja Award (to Martin Lövdén) of the Alexander von Humboldt Foundation donated by the German Federal Ministry for Education and Research (BMBF). The authors want to thank the following people for their important roles in conducting the COGITO study: Colin Bauer, Katja Müller-Helle, Birgit Heim, Annette RentzLühning, Werner Scholtysik, Julia Wolff, and a team of highly committed student research assistants.

Correspondence concerning this article should be addressed to Annette Brose, Max Planck Institute for Human Development, Center for Lifespan Psychology, Lentzeallee 94, 14195 Berlin, Germany. E-mail: brose@ mpib-berlin.mpg.de term variation in such phenomena has attracted attention for quite some time, the state-like qualities of cognitive performance have only recently moved into the focus of attention (e.g., Li, Huxhold, \& Schmiedek, 2004; Rabbitt, Osman, Moore, \& Stollery, 2001). The results of a few studies suggest that an individual's performance varies considerably and reliably across occasions (Nesselroade \& Salthouse, 2004; Rabbitt et al., 2001; Sliwinski, Smyth, Hofer, \& Stawski, 2006) - to the extent that this cannot be dismissed as noise and therefore calls for explanatory accounts (Nesselroade \& Salthouse, 2004).

This study took up the call to explain within-person variability ${ }^{1}$ in performance, with a focus on working memory (WM) performance. It investigated whether variability in WM performance from day to day was related to variability in negative affect, subjective control of attention, and motivation. Within-person relations will also be called couplings in the following. Our interest was inspired by quasi-experimental studies showing a relationship between these variables; in particular, studies on performance deficits in people with depressive symptoms that point to the role of attentional and motivational deficits (e.g., Ellis \& Ashbrook, 1988; Hertel \& Rude, 1991), and experimental studies manipulating emotions that show reliable effects on cognitive performance

\footnotetext{
${ }^{1}$ In the following, the term variability is used for within-person or intraindividual variation; the term variation is used for between-person variation or differences. When talking about variation more generally or when not being specific about whether we refer to variation at the withinor between-person level, we use the term variation.
} 
(e.g., Ellis, Moore, Varner, Ottaway, \& Becker, 1997; Richards \& Gross, 2000). Critical questions that emerged when applying this background to the question of correlates of day-to-day variability of WM within individuals were (a) whether more subtle day-today fluctuations of, for example, negative affect are meaningful enough to impact on performance, especially in comparison to between-person differences in depressive symptoms; and (b) whether more generally, the mechanisms that are discussed as underlying relationships between variables operate similarly at the between- and the within-person level of analysis. To elaborate, in this study we focused on associations between variables at the within-person level (e.g., intraindividual coupling of affect and performance), but as a background, we used theories and findings primarily discussed at the between-person level (e.g., covariation of depressive symptoms and performance across individuals).

\section{Day-to-Day Variability in Working Memory Performance}

The function of WM is to temporarily store and simultaneously manipulate information (Baddeley, 1986). Interindividual differences in WM capacity are related to the ability to control attention (Engle, 2002; see e.g., Oberauer, 2005, for other propositions regarding interindividual differences in WM). Day-to-day variability in WM is performance variation that can be differentiated from interindividual differences in performance levels, and from other types of within-person variability that occur on faster time scales (e.g., from trial to trial) or on slower timescales such as months or years. Trial-to-trial variability has been viewed as, for example, a marker of neurological integrity (e.g., Lövdén, Li, Shing, \& Lindenberger, 2007). One example of variability in cognition across months is strategy exploration in sensitive learning periods that can be observed in children acquiring math or language (Siegler, 1994).

Variables that have been studied in relation to performance variability on the timescale of days or weeks can be roughly divided into variables operating at the biological level (circadian rhythms, physical symptoms; Hasher, Zacks, \& May, 1999; Salthouse \& Berish, 2005) and variables operating at the psychological level (subjective representations of stress, motivation, and affect; Brose, Schmiedek, Lövdén, Molenaar, \& Lindenberger, 2010; Röcke, 2006; Salthouse \& Berish, 2005; Sliwinski et al., 2006). One impetus for studying within-person associations between these variables and performance, and for modeling cognitive variability, comes from the recognition that it "allows testing hypotheses regarding associations among cognitive processes as they transpire within individuals" (Sliwinski et al., 2006, p. 545). More generally, investigating associations within individuals across time allows the investigation of processes at the level at which they occur-the intraindividual level, for which findings at the interindividual level cannot provide a surrogate (Molenaar, 2004; Nesselroade, 1991). To illustrate, Sliwinski and colleagues (2006) found a negative within-person coupling between stress and WM performance. Attentional deficits on stressful days were discussed as a causal agent for the observed relation. Thus, the authors relied on central ideas about WM functioning, namely the central role that the ability to focus attention plays for interindividual differences (e.g., Engle, 2002) when explaining the within-person relation under investigation.
This study took a similar approach. We investigated negative affect, control of attention, and motivation as predictors of daily variability in WM because phenomenological accounts about each of the domains as well as theories on their relation lead to the expectation that they are functionally related within individuals across time. This has not, however, been thoroughly investigated.

\section{Variability in Working Memory and Negative Affect}

Various areas of research suggest that there is a relationship between affective experiences and how well individuals perform on cognitive tasks. A broad body of literature shows cognitive deficits in depressed and dysphoric individuals, in particular, in tasks requiring effortful processing such as episodic memory tasks or tasks measuring executive functions (Burt, Zembar, \& Niederehe, 1995; Harvey, 2004; Joormann, 2008; Rogers et al., 2004; but see e.g., Grant, Thase, \& Sweeney, 2001). In addition, experimental studies using mood induction paradigms found lower performance levels in groups which experience negative emotions (Ellis et al., 1997; Seibert \& Ellis, 1991). One explanation for these observations is based on a dual-task perspective. According to this perspective, a limited amount of cognitive resources can be allocated either to performance or to affective experiences-an idea explicated, for example, in the resource allocation model by Ellis and Ashbrook (1988). If a person experiences high negative affect, the emotion itself may capture attention, or attempts to regulate emotions are made, both of which place high demands on resources. Theories and empirical findings provide support for these ideas. For example, negative information including affect per se becomes the center of attention when people are depressed (Beck, 1967) - a phenomenon also known as ruminating about one's affective experiences (Nolen-Hoeksema, Wisco, \& Lyubomirsky, 2008). Rumination and biased attention to primarily negative information impair task performance (Lyubomirsky, Tucker, Caldwell, \& Berg, 1999; Siegle, Ingram, \& Matt, 2002). The idea that emotion regulation is cognitively costly found support in an experience sampling study (Riediger, Wrzus, Schmiedek, Wagner, \& Lindenberger, 2011) and with experimental paradigms that manipulate emotion regulation and measure its effects on cognitive performance. In particular, suppression of emotion and downregulation of negative emotions was inversely related to cognitive performance in younger adults (Richards \& Gross, 2000; Scheibe \& Blanchard-Fields, 2009; Shamosh \& Gray, 2007).

A second explanation for a relation between affect and cognition focuses on motivational aspects of behavior. A lack of initiative when experiencing aversive states may be responsible for performance deficits. Again, clinical research on depression, but also experimental investigations, support this idea (Hertel, 2000; Hertel \& Hardin, 1990; Hertel \& Rude, 1991; Kuhl \& Helle, 1986). One study that tested the role of initiative in task performance revealed that a lack of initiative rather than limited attentional resources may be the better explanation for deficits in depressed as compared to nondepressed subjects (Hertel \& Rude, 1991). In this study, learning tasks varied regarding the extent to which the focus of attention was constrained. In the more constrained condition, performance of the two groups did not differ. Hertel and Rude (1991) concluded that people with depression did not perform poorly because resources were not available, but because of a deficit in the initiative to allocate resources to the task. 
This raises the question of whether the above considerations imply that days with varying levels of negative affect are days with reduced performance in WM. The intensity of experiencing negative affect when depressed or after an experimental induction probably exceeds the fluctuations in mood in daily life, and mechanisms proposed to link affect and cognition may only become relevant once a particular threshold of experiencing negative affect is reached (Ellis \& Ashbrook, 1988). For example, regulatory effort may only be elicited under such a circumstance. In addition, viewing depression as partly being caused by stable dispositions such as reduced or inadequate cognitive control of negative information (Beevers, 2005; Joormann, 2008) may challenge the idea that the mechanisms can be transferred to everyday functioning of normal populations.

Despite this, phenomenological accounts on affective functioning suggest that the mechanisms that are assumed to link affect and cognition operate constantly within individuals across time. Next to other functions, affective states are assumed to indicate discrepancies from desired outcomes, including for example, goals or affective states per se (Carver \& Scheier, 1999; Larsen, 2000). The latter point has also been emphasized by the mood-behavior model (Gendolla, 2000), according to which behavior is partly guided by hedonic motives. Thus, affect is monitored and is part of a control system that regulates and initiates behavior in order to minimize discrepancies from optimal states (Carver \& Scheier, 1999). The notions that affect captures attention, is target of regulation, and elicits appraisal of situations and regulation effort such as reappraising of situations all seem to imply the use of resources, some of which seem to be cognitive in nature (attention directed at affect), and others motivational (effort made to repair mood).

Three studies have already investigated daily fluctuations in negative affect as a within-person correate of varying cognitive performance levels. According to two of those, there is no evidence of a coupling of affect and cognitive performance across occasions (within days, Salthouse \& Berish, 2005; across days, Sliwinski et al., 2006). However, both studies focused on RT as performance outcome, not on accuracy. The third study that investigated couplings between negative affect and accuracy of performance found lower accuracy on days with more negative mood (Riediger et al., 2011).

In sum, interindividual differences in affect-cognition relationships and intraindividual processes of affective functioning refer to similar principles: affect per se captures attention, affect regulation is resource demanding, and once affect is perturbed, repair attempts are made-potentially resulting in performance deficits because of attentional or motivational deficits. We, therefore, assume that couplings between daily variability in negative affect and WM will be found.

\section{Variability in Working Memory and Control of Attention}

The ability to control attention is a trait-like characteristic, and a discriminating feature between individuals who differ in WM capacity. Higher levels of attentional control are associated with higher levels of WM, and some authors see this ability as a core feature of WM (Engle, 2002; Kane, Conway, Hambrick, \& Engle, 2007). This ability may at least partly be consciously accessible. For instance, Kane and colleagues (Kane, Brown, Little, \& Silvia,
2007) found that self-reported levels of mind wandering are correlated with WM capacity at the between-person level. Also, interindividual differences in people's self-reported dispositional tendency to experience task-irrelevant thoughts are associated with cognitive performance levels (Sarason, Sarason, Keefe, Hayes, \& Shearin, 1986).

Does the ability to control attention vary from day to day and can such variation predict variation in WM? As described in the context of negative affect, whether attention is directed at affective experiences may fluctuate across time, depending on whether a state requires regulation. In addition, as was proposed by Sliwinski and colleagues (2006), daily stressors may capture attention from time to time. Both these factors, and plausibly others such as health problems or even positive events, may reduce attentional resources available for performance on cognitively demanding tasks. Thus, it seems plausible that control of attention required for task performance not only varies across individuals, but that it has a state-like component. The latter is likely to be related to performance. In particular, less control should result in reduced performance. The present study examined this using a self-report measure of control of attention. In addition, we expected that the variability in negative affect that is related to WM performance could be partly predicted by variations in control of attention. This assumption reflects the proposition that a perturbation of affect captures attention and elicits regulation that may be cognitively effortful, as described in the previous section.

\section{Variability in Working Memory and Motivation}

The relationship between motivation and performance is well documented. This relationship exists at both the level of traits and at the level of states (e.g., interindividual differences in achievement motivation are related to performance variation; Marsh, Byrne, \& Shavelson, 1988; intraindividual variability in effort is related to performance variability; Yeo \& Neal, 2004). Various mechanisms are discussed as underlying the relationship (for a summary, see Eccles \& Wigfield, 2002). For example, high levels of motivation may result in persistence (Ryan \& Deci, 2000) or interference control (Kuhl, 1987). Increasingly, these ideas are approached and supported at the within-person level (e.g., Yeo \& Neal, 2004). In line with these ideas, we have reported that effort and enjoyment are related to performance accuracy across days in the N-back task used in the current study (Brose et al., 2010). We held tasks and laboratory conditions constant across days. Thus, variability in motivation was ascribed to varying physical and mental conditions as well as situational demands outside the laboratory on a particular study day.

With this background, we expected that a global measure of task-related motivation would also predict WM performance across days. Days with reduced motivation were predicted to be days with reduced performance. We extended the previous study by investigating the coupling of motivation with both accuracy and RT. In addition, we examined whether the above proposition - that motivation is reduced on days with high negative affect-finds support. Such a finding would be in line with the idea that one reason why performance suffers on days when mood is negatively affected is a lack of initiative or motivation, perhaps because a more important goal on such a day is to repair the mood (see section on affect-WM coupling). 


\section{Summary of the Current Study}

This study investigated whether day-to-day variability in WM performance was associated with variability in experiencing negative affect and with varying levels in control of attention and motivation. We expected decreased performance levels on days with increased negative affect and on days with decreased control of attention and motivation. That is, we assumed we would find associations among variables within individuals from day to day. Based on two major propositions in the literature as to why negative affective experiences are associated with performance levels, namely a resource allocation account and a motivational account, we further examined whether there was evidence in our study that supports such propositions. In particular, by means of multilevel regression analyses, we tested whether affect-related performance variations can partly be predicted by variations in control of attention and motivation. As this study was correlational in nature, the relationships among predictors that were hypothesized and tested did not allow causal inferences (cf. Lindenberger \& Potter, 1998).

\section{Method}

The current investigation is part of a larger study, the COGITO Study, conducted at the Center for Lifespan Psychology, Max Planck Institute for Human Development, Berlin (Schmiedek, Lövdén, \& Lindenberger, 2009; 2010). The COGITO Study follows a pretest-posttest control group design, with a microlongitudinal study phase of 100 days in the experimental group at its core. The present study reports data from this longitudinal phase.

\section{Participants and Procedure}

This study included 101 younger participants (51.5\% women, age: $20-31, M=25.6, S D=2.7)$. On average, participants had 12.5 years of high school education; $98 \%$ were single and $2 \%$ were married; the majority was university students $(67 \%)$ and the others were employed (13\%), unemployed $(11 \%)$, students (8\%), or apprentices $(1 \%)$. One sample characteristic that could have possible influenced the results is depression status as assessed by the prevalence of depressive symptoms. Such an influence is likely because the link between affect and cognitive performance through control of attention may be more pronounced among more depressed individuals (Joormann, 2008). We evaluated depressive symptoms in our sample with a German version of the CES-D (Hautzinger, 1988). According to confidence intervals, our sample did not differ from a German representative sample (Hautzinger \& Bailer, 1993), (95\%-CI $\mathrm{I}_{\text {repr_sample }}[13.74,14.86]$; $95 \%-\mathrm{CI}_{\text {this_study }}$ $[13.69,17.09])$. Thus, the present sample was not atypical in relation to the frequency of reported depressive symptoms.

Participants were recruited through newspaper advertisements, word-of-mouth recommendations, flyers distributed in university buildings, community organizations, and local stores. The COGITO Study advertisement addressed individuals interested in working on cognitive tasks for 4-6 days a week for a period of about six months. The recruitment steps for participants in the study were: (a) a telephone interview: people were given information about the study and they were asked whether the requirements for study participation such as time investment could be met; (b) a 1-hr group session: potential candidates for participation were invited to attend a session where aims of the study were explained, information on incentives was given, and the digit-symbol substitution test and a questionnaire on sociodemographic variables were administered. After this session, individuals could sign up for the study. Study participation began and ended in group sessions with 10 days of pre- and posttests (2-2.5 hours). The microlongitudinal phase (100 sessions, 1-1.5 hours each) was scheduled on an individual basis. During the microlongitudinal study phase, participants worked on the tasks individually in rooms with three to six computers. The total number of sessions per person in the microlongitudinal phase ranged from 87 to $107(M=100)$.

Attrition occurred at different phases. Seven participants withdrew during the pretest, and 19 participants dropped out during the 100 daily sessions (for details on dropouts, see Schmiedek, Bauer, Lövdén, Brose, \& Lindenberger, 2010). Incentives for study participation varied between 1450 and 1950 Euros. A bonus system was implemented to reduce attrition and to motivate participants to complete the study as quickly as possible.

The daily sessions were carried out from Monday to Saturday between 8 a.m. and 7.30 p.m. Each assessment started with a visual acuity task, followed by computerized self-reports (5-8 minutes) and 12 different computerized cognitive tasks (40-65 minutes): three WM tasks, three episodic memory tasks, and six perceptual speed tasks. The sessions concluded with self-report (1-2 minutes). The laboratory was equipped with 32 identical PCs and monitors. The order of the cognitive tasks was invariant. The stimuli for the cognitive tasks were chosen from a pool of stimuli according to algorithms that varied from task to task.

\section{Measures}

The answering format of the following self-report scales was identical. Subjects were asked to indicate on 8-point scales ranging from 0 (does not apply at all) to 7 (applies very well) how well items represented their states.

Negative affect. Daily affect was assessed with a German version of the 20-item state version of the Positive and Negative Affect Schedule (PANAS; Watson, Clark, \& Tellegen, 1988). Items were presented in random order, varying across days. For the present study, the 10-item negative affect subscale is relevant. Subjects were asked to rate how well adjectives such as distressed or afraid described their current mood. Five items were excluded from further analysis (guilty, scared, hostile, ashamed, afraid) because $8 \%$ to $16 \%$ (depending on item) of the participants did not fluctuate at all on them $(S D=0)$. The internal consistency of the scale with the remaining items (distressed, jittery, nervous, upset, irritable) was on average .85, ranging from .75 to .92 in Sessions 1 to 100 . The average score across the five items was used in the analyses.

Subjective control of attention. Subjective control of attention as the evaluation of one's ability to concentrate on tasks was measured with two items from the German version of the SelfRegulation scale (Schwarzer, Diehl, \& Schmitz, 1999). To capture daily fluctuations, these statements were worded slightly differently than the original items, "Today, I can concentrate on one activity for a long time if necessary" and "Today, I can control my thoughts from distracting me from the task at hand". The average correlation of the two items was .80 , ranging from .60 to .90 in 
Sessions 1 to 100 . The average of the two items was used in the analyses.

Motivation. Motivation as investigated in this study was conceptualized as the global motivation to work on the cognitive tasks on a particular day. Prior to working on the cognitive tasks, participants were asked to indicate how well the statement "I am highly motivated to work on the cognitive tasks today" reflected their attitude.

Working memory: 3-back task. A 3-back task measured spatial-figural WM. A sequence of 39 black dots appeared at varying locations in a 4 by 4 grid. Participants had to respond to each dot as to whether it was in the same position as the dot three steps earlier in the sequence or not. Responses were given via button press with one finger of the left and right hand. This task places high demands on serial attention. The dots appeared at random locations in the grid, with the constraints that (a) 12 items were targets; (b) dots did not appear in the same location in consecutive steps; and (c) exactly three items each were 2-, 4-, 5-, or 6-back lures, that is, items that appeared in the same position as the items 2, 4, 5, or 6 steps earlier. Presentation rates for the dots were individually adjusted based on pretest performance, and were kept constant within individuals throughout the daily testing phase. Presentation time was $500 \mathrm{~ms}$. Interstimulus intervals were 500, 1500 , and $2500 \mathrm{~ms}$, depending on pretest performance. In each daily session, four trials were included. Accuracy and RT scores were averaged across the four different trials on all days and these values were used for analyses. Because presentation times were individually adjusted, the mean level of the dependent variables and its predictors were not further investigated.

\section{Statistical Analyses}

Reliability of day-to-day variability in working memory performance. When testing our hypotheses about couplings between cognitive performance and, for example, negative affect within individuals across days (see below), it makes a difference how systematic a given individual's day-to-day fluctuations are. The more systematic the observed fluctuations are, the more likely it is that meaningful correlates are present and observable. Put differently, only to the degree that participants vary systematically from one day to the other can one expect to be able to predict this variability. As there is rather meager evidence on whether WM actually varies systematically from day to day and interindividual differences therein, this study identified systematic daily variability in WM performance and took results into consideration when testing hypotheses, as we will now describe.

Performance variability within individuals as measured in this study across 100 days had various sources (see Figure 1). A training gain can be expected, as can day-to-day variation around this average trend, and variation across trials (consider that the 3-back task was measured in four trials each day). We designate these three variance components as training gain, day-to-day variance $\left(\sigma_{\text {days }}^{2}\right)$, and trial-to-trial variance $\left(\sigma_{\text {trials }}^{2}\right)$. The variance we aim to explain in this study is day-to-day variance. Following Wittmann's (1988) multivariate reliability theory, one may consider this portion of the total variance as wanted variance. According to Wittmann (1988), this term implies an evaluation of variance components not in the sense of measurement quality but in the sense of theory-guided distinctions.

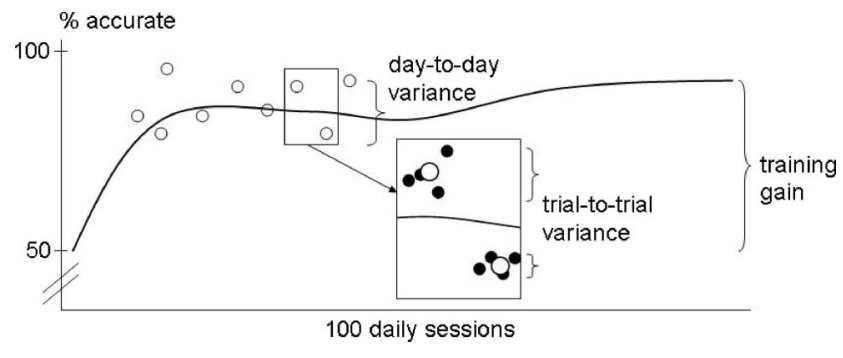

Figure 1. Variance components of an individual's task performance (accuracy, 3-back task) across 100 days: training gain, day-to-day variance (open circles), and trial-to-trial variance (filled circles).

The first step in identifying systematic day-to-day variation was to model training gains (see below), resulting in predicted performance scores. From this, it follows that observed scores vary around predicted scores, named observed day-to-day variance. Ideally, performance on the 3-back task would be measured perfectly reliably within days $\left(\sigma_{\text {trials }}^{2}\right)$, so that all variation observed across days $\left(\sigma_{\text {days }}^{2}\right)$ is systematic or reliable. With a large number of observations per day, such a perfectly reliable performance score could theoretically be achieved. However, due to constraints in the experimental situation, the number of observations is limited, and due to various sources of variation, such as interactions between a participant and a stimulus or measurement error, scores differ from one trial to the next. Thus, systematic or unsystematic variability from trial-to-trial contributes to observed variance across days, observed $\sigma_{\text {days }}^{2}=$ reliable (systematic) $\sigma_{\text {days }}^{2}+\sigma_{\text {trials }}^{2} / n_{\text {trials }}$. Once the observed day-to-day variance is decomposed into these two contributing components (this can be achieved by fitting unconditional multilevel models with, e.g., SAS PROC MIXED; Singer \& Willet, 2003), for each individual, a score that characterizes the amount of systematic (i.e., reliable) daily variance (DailyVar) can be derived using the following formula

$$
\text { DailyVar }=\sigma_{\text {days }}^{2} /\left[\sigma_{\text {days }}^{2}+\left(\sigma_{\text {trials }}^{2} / n_{\text {trials }}\right)\right] .
$$

DailyVar quantifies the relative magnitude of the day-level variance component (an individual's observed variance across 100 days, $\sigma_{\text {days }}^{2}$ ) and the trial-level variance component (an individual's variance of the odd and even trials divided by $2, \sigma_{\text {trials }}^{2} / n_{\text {trials }}$ ) that were estimated at the level of individuals. Thus, DailyVar is the proportion of the total variance that is systematic or reliable day-to-day variance. As DailyVar is estimated for every individual, it has the status of an attribute of an individual and it results in a distribution of coefficients indicating how systematic each individual's performance varied across days. Figure 2 illustrates interindividual differences in DailyVar-showing that interindividual differences in the reliability of day-to-day fluctuations were impressively large. Some individual's performance varied rather systematically from day to day. However, the selective contribution of systematic day-to-day variability to observed day-to-day variability was close to zero in other individuals, indicating a large contribution of trial-to-trial variability to observed variability. The average amount of reliable daily variability (DailyVar) in accuracy was $36 \%$. Reaction times fluctuated more systematically from day to day; the average DailyVar was $68 \%$.

When testing our hypothesis on, for example, WM-affect couplings, we included information on how systematically individuals 


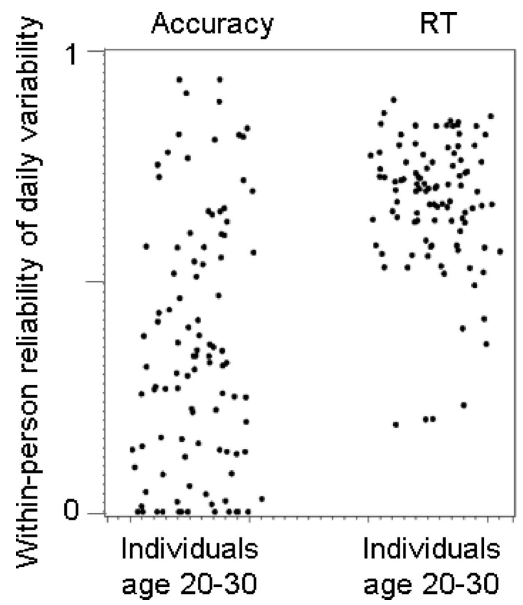

Figure 2. 3-back task, accuracy and RT: Proportion of observed day-today variance that is systematic/reliable day-to-day variance (reliability of daily variability, DailyVar), estimated for each individual (represented by one dot).

varied from one day to the next by including DailyVar as a between-person (level-2) predictor. Couplings tested were assumed to be stronger the more systematic variation was from day to day (see below).

Testing research hypotheses. The research questions were investigated using a mixed model approach to account for the hierarchical structure of the data. The analyses were conducted using SAS PROC GLIMMIX. This procedure was chosen because it allows the modeling of complex changes across the study time (see below). In addition, research questions that follow the logic of multilevel regression can be approached with this procedure in a similar fashion as if they were tested with more common multilevel modeling statistical tools such as SAS PROC MIXED. We considered two levels when answering the research questions. The lower level represented occasions or "days" completed by individuals, who, in turn, represented the upper level in our models. Variables that were collected on a daily basis are time-varying, level-1 variables. Characteristics of the participants that are stable (in a conceptual sense) were level-2 variables. Including predictors of performance (e.g., negative affect) in models resulted in a coefficient for an average individual (e.g., fixed effects) as well as in interindividual variation around these coefficients (e.g., random effects). The same basic model served to answer all research questions (affect serves as an example in the following):

$$
\text { Level-1 model: } D V_{i j}=\beta_{0 i}+\beta_{1 i}\left(\text { Days }_{i j}\right)+\beta_{2 i}\left(\text { Affect }_{i j}\right)+r_{i j}
$$

$$
\begin{aligned}
\text { Level-2 model: } & \beta_{0 i}=\gamma_{00}+\gamma_{01}\left(\text { DailyVar }_{0 i}\right)+u_{0 i} \\
& \beta_{1 i}=\gamma_{10}+u_{1 i} \\
& \beta_{2 i}=\gamma_{20}+\gamma_{21}\left(\text { DailyVar }_{0 i}\right)+u_{2 i} .
\end{aligned}
$$

To paraphrase the equations: Performance (DV: accuracy or $\mathrm{RT}$ ) of person $\mathrm{i}$ on occasion $\mathrm{j}$ is the criterion variable; it is predicted by person i's mean performance (with regression coefficient $\beta_{0 i}$ ), a component representing person i's change across time (with $\beta_{1 i}$ ), and concurrent negative affect (with $\beta_{2 i}$ ). The parameter $r_{i j}$ is person i's residual deviation at occasion j. $\beta_{\mathrm{O} i}$ is decomposed into an intercept term $\left(\gamma_{00}\right)$, an effect of the personlevel variable DailyVar $\left(\gamma_{01}\right)$, which indicates how reliably participants' performance varied from day to day, and a random effect $u_{0 i} ; \beta_{2 i}$ is decomposed into an intercept term $\left(\gamma_{20}\right)$, a moderating effect of the person-level variable DailyVar $\left(\gamma_{21}\right)$, and a random effect $u_{2 i}$. The random effects denote individuals' deviations from the fixed effects. The fixed effect referring to days ( $\beta_{1 i}$, change across time), also has a random coefficient $\left(u_{1 i}\right.$, see below). In short, performance is thought to change across the study time and to covary with affect; with the size of this coupling being moderated by how systematically individuals fluctuate in performance from day to day.

Removing trends across study time. Trends in cognitive performance across the study duration were modeled with penalized radial spline smoothing functions as implemented in SAS PROC GLIMMIX (Ruppert, Wand, \& Carroll, 2003; for an earlier application of this method to data from the COGITO Study, see also Schmiedek et al., 2009). The choice of this smoothing technique was chosen because for several individuals, improvements due to practice could not be sufficiently well captured with theoretically expected learning curves such as exponential or power functions. As a semiparametric method, penalized radial spline smoothing functions fit each individual's time series simultaneously using a mixed model approach, with individual differences in the functions captured by random effect parameters. In this way, the amount of smoothing is the same for all individuals. Predicted values can be created using best linear unbiased prediction methods (Ruppert et al., 2003). Visual inspection of these predicted trends together with the observed data indicated that even the most extreme patterns of intraindividual change were captured sufficiently well with this approach. Figure 3 illustrates the results of modeling mean trends in performance with spline smoothing by presenting observed scores and predicted curves for two individuals.

This procedure was also used to correct for larger changes across study time in predictor variables (negative affect, attentional control, and motivation). Such changes could be observed primarily in motivation, mostly as some form of discontinuous decline. Regressing these predictor variables on time using SAS PROC GLIMMIX resulted in residuals that fluctuated around an average trend. These residuals were used when testing our hypotheses, and they correlated highly (average $r_{\text {negative affect }}=.89$; average $r_{\text {attention }}=.91$; average $r_{\text {motivation }}=.89$ ) with values derived

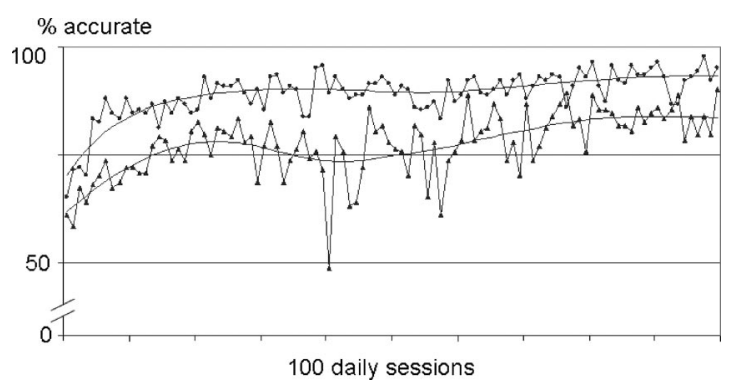

Figure 3. Two individuals' accuracy across 100 days: Results from modeling mean trends in performance with spline smoothing (observed scores and predicted curves). 
from group mean centering, the common treatment of lowerlevel variables in multilevel modeling (Singer \& Willett, 2003).

\section{Results}

\section{Average Variability and Reliability of Day-to-Day Variability}

Results from descriptive analyses of the self-report data and from the WM task are summarized in Table 1. All these descriptive statistics are based on residuals after controlling for trends across study time as we were interested in the variation from day to day, and not in predicting longer-term changes such as practice-related improvements. Individuals varied from day to day on all study variables as becomes apparent when observing the intraindividual $S D$ (iSD, Table 1). That is, negative affect, subjective control of attention (abbreviated CoA in the Results section), motivation, and accuracy and RT of N-back performance were not stable, but fluctuated from day to day. In addition, individuals differed from each other regarding how much they fluctuated from day to day (distribution of the iSD). Some individuals were more stable, others varied more. In addition to calculating the iSD, we estimated the relative amount of within- and between-person variability in selfreport variables by means of the intraclass correlation (ICC; see e.g., Singer \& Willett, 2003). Results are illustrated in Figure 4. From the total amount of variation in negative affect, $36 \%$ is variability across time (within-person) and the remaining $64 \%$ is variation between individuals (between-person). Similarly, $46 \%$ of variation in CoA is within-person variation, and $42 \%$ of variation in motivation is withinperson variation. Table 1 also provides information on how the self-report variables relate at the within-person level, that is, on the mean and the $S D$ of the intraindividual correlations.

\section{Couplings of WM With Affect, Attention, and Motivation: Bivariate Analyses}

In the following, day-to-day bivariate couplings between N-back performance (accuracy and RT) and negative affect, CoA, and motivation are presented. For two reasons, presentation of results focuses on the parameters representing couplings (i.e., average performance and training gains are not the focus of attention here). First, the average level of performance depends on

Table 1

Means and SD of Intraindividual Variability (iSD) in SelfReport Variables and Working Memory Performance and of Intraindividual Correlations of Self-Report Variables

\begin{tabular}{lccc}
\hline & $\begin{array}{c}\text { Average } \\
\text { iSD (SD) }\end{array}$ & \multicolumn{2}{c}{ Correlations (SD) } \\
\hline Self report & & $(2)$ & $(3)$ \\
$\quad$ Negative affect (1) & $0.71(0.31)$ & $-.24(.18)$ & $-.25(.15)$ \\
$\quad$ Control of attention (2) & $0.83(0.29)$ & & $.43(.19)$ \\
$\quad$ Motivation (3) & $0.99(0.33)$ & & \\
Working memory & & & \\
$\quad$ Accuracy (\%) & $4.3(3.1)$ & \\
$\quad$ RT (ms) & $29.14(24.96)$ & \\
\hline
\end{tabular}

Note. Coefficients of self-report variables can be interpreted in scale point units (8-point answering scale, $0-7$ ).

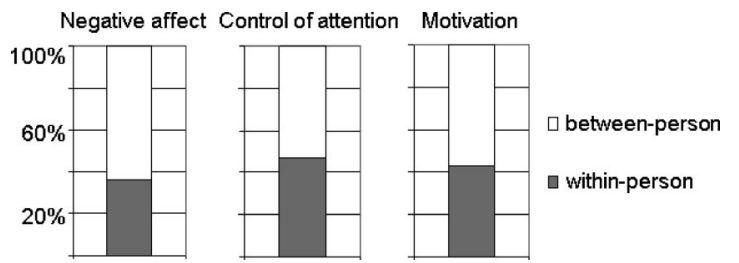

Figure 4. Negative affect, control of attention, and motivation: Relative amount of variation at the within- and between-person level.

presentation times that were individually adapted. Second, reporting the various estimates representing change in performance across study time was deemed unnecessary, given that in this paper, we focus on day-to-day fluctuation. Particular attention will be paid to the interaction term in the analyses (interaction of predictor with DailyVar) because reliable day-to-day variance is considered to be a precondition for testing the hypotheses under investigation.

We expected that days with more negative affect, with reduced $\mathrm{CoA}$, and with reduced motivation were days with poorer N-back performance. We explored whether both indicators of performance, accuracy and RT, were equally affected. Results are presented in Table 2, with further illustration in Figure 5. Regarding accuracy, our expectations were confirmed. Accuracy on the $\mathrm{N}$-back task was significantly predicted by the interaction between negative affect and DailyVar, $F(1,8,283)=6.08, p<.05$. This means that individuals who fluctuated systematically from day to day in accuracy on the N-back task were less accurate on days with high negative affect. To illustrate: For a hypothetical individual whose performance variation from day to day would be perfectly systematic (DailyVar $=1$ ), experiencing negative affect that is 0.71 scale point units above average $(0.71$ represents the average intraindividual $S D$ found in negative affect across 100 days, see Table 1) would be associated with a decrease in accuracy of $1.1 \%$ which is about one fourth of the average amount of within-person variation in accuracy (average iSD, see Table 1). In addition, a Pseudo- $\mathrm{R}^{2}$ statistic is reported in Table 2. It represents the reduction of unexplained within-person variation in accuracy. It was derived by comparing the model presented in Table 2 with a model without any level-1 predictors except for time (study days). That is, it was derived by calculating the difference in residual variation of the two models (Singer \& Willett, 2003). The Pseudo- $\mathrm{R}^{2}$ statistic shows that $2 \%$ of day-to-day variation in performance can be explained by daily fluctuations in negative affect.

Similarly, accuracy on the N-back task was significantly predicted by the interaction between CoA and DailyVar, $F(1$, $8,282)=19.77, p<.05$, and by the interaction between motivation and DailyVar, $F(1,8,281)=27.93, p<.05$. Lower values of accuracy were associated with lower values of CoA and lower values of motivation. The Pseudo- $\mathrm{R}^{2}$ statistics presented in Table 3 suggest that daily variation in CoA explains 3.5\% of variation in accuracy and that daily variation in motivation explains $8 \%$ of variation in accuracy. In sum, day-to-day variations in negative affect, $\mathrm{CoA}$, and motivation covaried with day-to-day fluctuations in accuracy of performance.

In contrast to accuracy, daily variations in RT were not predicted by daily variations in negative affect and $\mathrm{CoA}$, that is, by interactions of these predictors with DailyVar, $F(1,8,180)=0.05$, 
Table 2

Bivariate Couplings Between Performance on the 3-Back Task (Accuracy and RT) and Daily Negative Affect, Control of Attention, and Motivation

\begin{tabular}{|c|c|c|c|c|c|c|c|c|c|c|c|c|}
\hline & \multicolumn{4}{|c|}{ Predictor negative affect } & \multicolumn{4}{|c|}{ Predictor control of attention } & \multicolumn{4}{|c|}{ Predictor motivation } \\
\hline & \multicolumn{2}{|l|}{ ACC } & \multicolumn{2}{|l|}{$\mathrm{RT}$} & \multicolumn{2}{|l|}{ ACC } & \multicolumn{2}{|l|}{$\mathrm{RT}$} & \multicolumn{2}{|l|}{$\mathrm{ACC}$} & \multicolumn{2}{|l|}{ RT } \\
\hline & Coefficient & $S E$ & Coefficient & $S E$ & Coefficient & $S E$ & Coefficient & $S E$ & Coefficient & $S E$ & Coefficient & $S E$ \\
\hline \multicolumn{13}{|l|}{ Fixed effects } \\
\hline Intercept & $2.3^{*}$ & 0.19 & 312.7 & 48 & $2.3^{*}$ & 0.19 & 312.7 & 48 & $2.3^{*}$ & 0.19 & 312.7 & 49 \\
\hline DailyVar & $-0.72^{*}$ & 0.21 & 23.3 & 68 & $-0.72 *$ & 0.22 & 23.3 & 68 & $-0.71^{*}$ & 0.22 & 23.3 & 69 \\
\hline Level-1 predictor & -0.01 & 0.02 & 1.1 & 3.7 & -0.01 & 0.02 & 4.1 & 3.8 & -0.02 & 0.02 & $6.2^{*}$ & 2.7 \\
\hline Level-1 predictor $*$ DailyVar & $-0.11^{*}$ & 0.05 & 1.2 & 5.3 & $0.20^{*}$ & 0.05 & -5.8 & 5.4 & $0.23^{*}$ & 0.04 & $-9.7^{*}$ & 3.9 \\
\hline \multicolumn{13}{|l|}{ Random coefficients } \\
\hline Level-1 predictor & \multirow{2}{*}{\multicolumn{2}{|c|}{0.008023}} & \multicolumn{2}{|c|}{$6.18 \mathrm{E}-6$} & \multirow{2}{*}{\multicolumn{2}{|c|}{0.009425}} & \multicolumn{2}{|c|}{0.00002} & \multicolumn{2}{|c|}{0.008896} & \multicolumn{2}{|c|}{$2.431 \mathrm{E}-6$} \\
\hline Residual & & & \multirow{2}{*}{\multicolumn{2}{|c|}{$\begin{array}{l}0.0018 \\
0.11 \%\end{array}$}} & & & \multirow{2}{*}{\multicolumn{2}{|c|}{$\begin{array}{l}0.0018 \\
0.55 \%\end{array}$}} & \multirow{2}{*}{\multicolumn{2}{|c|}{$\begin{array}{l}0.002736 \\
8.00 \%\end{array}$}} & \multirow{2}{*}{\multicolumn{2}{|c|}{0.0018}} \\
\hline Pseudo-R ${ }^{2}$ (Level-1) & \multicolumn{2}{|c|}{$2.00 \%$} & & & \multicolumn{2}{|c|}{$\begin{array}{l}0.002859 \\
3.50 \%\end{array}$} & & & & & & \\
\hline
\end{tabular}

${ }^{*} p<.05$. Additional fixed effect not listed: session; variance component not listed: variance session (smoother).

$p>.05$, and $F(1,8,181)=1.18, p>.05$, respectively. Thus, for N-back performance fluctuations regarding RT, it did not matter whether individuals were high or low in negative affect or subjective attentional control. In contrast, in individuals who varied systematically from day to day, reduced motivation was associated with increased RTs, $F(1,8,179)=5.92, p<.05$. Thus, individuals were slower on the N-back task on days when they were less motivated. However, the variance explained by motivation was very small $(0.11 \%)$.

\section{Couplings of WM With Affect, Attention, and Motivation: Combined Analyses}

The following results are concerned with how the different predictors of N-back performance are related. Two possible scenarios were described regarding how the predictors of performance may be related (negative affect is related to CoA, both are related to performance; negative affect is related to motivation, both are

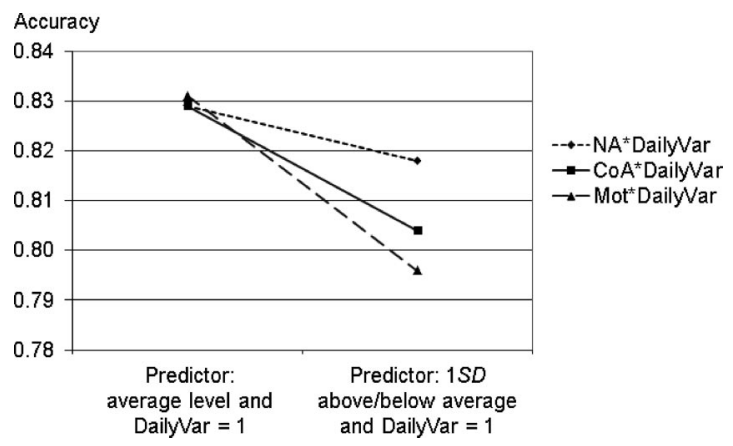

Figure 5. Illustration of the bivariate couplings between accuracy and negative affect (NA), control of attention (CoA), and motivation (Mot). In particular, the interaction of the different predictors with the level-2 variable representing reliability of daily variability (DailyVar) is depicted; values chosen to represent the coupling between accuracy and the predictors are DailyVar $=1$ and predictor $=1$ average intraindividual $S D$ different from average (above in the case of NA; below in the case of CoA and Mot) related to performance). It was hypothesized that a portion of the covariance between negative affect and performance can be predicted by variations in $\mathrm{CoA}$ and motivation. As was explained in the introduction, it is possible that both variables actually play a causal role in the relation between negative affect and performance, that is, they (partly) mediate the effect of negative affect on cognition. As this study is correlational in nature, however, we refrain from making causal inferences and from describing the following results in terms of mediation analyses.

To investigate whether the coupling between negative affect and $\mathrm{N}$-back performance can partly be predicted by variations in CoA, the above model testing the affect-performance relation was extended. The additional level-1 predictor CoA and also the interaction term CoA x DailyVar were added to Equation 2 presented in the previous section. The predictor CoA was allowed to vary across individuals, represented by an additional variance component, $u_{3 i}$. The analyses were restricted to accuracy of performance as the results presented above showed that the predictors of interest in this study were only coupled with accuracy but not with RT. The results are presented in Table 3, Model 1 . Whereas in this model, the interaction term CoA $\mathrm{x}$ DailyVar was significantly related to accuracy in WM performance, $F(1,8,181)=22.36, p<.05$, the interaction term negative affect $x$ DailyVar that was a significant predictor of $\mathrm{N}$-back performance in the bivariate analysis was no longer significant, $F(1,8,181)=1.15, p>.05$. Thus, when taking the two predictors $\mathrm{CoA}$ and negative affect into consideration simultaneously, it was revealed that they shared predictive variance regarding accuracy. The total amount of within-person variance explained was $4.5 \%$. Both predictors are related to performance variation, but the variance in negative affect related to performance is not unique, but can be predicted by variation in CoA.

Similarly, we tested whether the coupling between negative affect and N-back performance can partly be predicted by variations in motivation (Table 3, Model 2). The interaction term motivation x DailyVar was a significant predictor of accuracy, $F(1,8,179)=31.21, p<.05$, but, unlike in the bivariate analysis, the interaction term negative affect $\mathrm{x}$ Daily Var was not significant, $F(1,8,179)=0.55, p>.05$. The total amount of within-person 
Table 3

Negative Affect, Control of Attention, and Motivation Predicting Performance in 3-Back Task (Accuracy): Combined Analyses

\begin{tabular}{|c|c|c|c|c|c|c|}
\hline & \multicolumn{2}{|c|}{ Model 1} & \multicolumn{2}{|c|}{ Model 2} & \multicolumn{2}{|c|}{ Model 3} \\
\hline & Coefficient & $S E$ & Coefficient & $S E$ & Coefficient & $S E$ \\
\hline \multicolumn{7}{|l|}{ Fixed effects } \\
\hline Intercept & $2.3^{*}$ & 0.19 & $2.3^{*}$ & 0.19 & $2.3^{*}$ & 0.19 \\
\hline DailyVar & $-0.72^{*}$ & 0.22 & $-0.70^{*}$ & 0.22 & $-0.69^{*}$ & 0.22 \\
\hline Negative affect & -0.004 & 0.02 & 0.002 & 0.02 & -0.01 & 0.02 \\
\hline Negative affect*DailyVar & -0.05 & 0.04 & -0.03 & 0.04 & -0.01 & 0.04 \\
\hline Control of attention & -0.03 & 0.02 & & & -0.02 & 0.02 \\
\hline Control of attention $*$ DailyVar & $0.20^{*}$ & 0.04 & & & $0.09^{*}$ & 0.04 \\
\hline Motivation & & & -0.02 & 0.02 & -0.01 & 0.02 \\
\hline Motivation * DailyVar & & & $0.23^{*}$ & 0.04 & $0.20^{*}$ & 0.04 \\
\hline \multicolumn{7}{|l|}{ Random coefficients } \\
\hline Negative affect & \multicolumn{2}{|c|}{$\begin{array}{l}0.00625 \\
0.00729\end{array}$} & \multicolumn{2}{|c|}{0.00648} & \multicolumn{2}{|c|}{0.00475} \\
\hline Control of attention & \multirow{2}{*}{\multicolumn{2}{|c|}{0.00729}} & & & \multicolumn{2}{|c|}{0.00167} \\
\hline Motivation & & & \multicolumn{2}{|c|}{0.00758} & \multicolumn{2}{|c|}{0.00762} \\
\hline Residual & \multicolumn{2}{|c|}{0.00283} & \multicolumn{2}{|c|}{0.00270} & \multirow{2}{*}{\multicolumn{2}{|c|}{$\begin{array}{l}0.0026 \% \\
9.50 \%\end{array}$}} \\
\hline Pseudo- $\mathrm{R}^{2}$ (Level-1) & \multicolumn{2}{|c|}{$4.50 \%$} & \multicolumn{2}{|c|}{$9.00 \%$} & & \\
\hline
\end{tabular}

${ }^{*} p<.05$. Additional fixed effect not listed: session; variance component not listed: variance session (smoother).

variation explained by this model is $9.0 \%$. This result again reveals that some portion of the relation between negative affect and accuracy can be predicted by variation in motivation.

We followed up the last two results with a model that tested all predictor variables of accuracy (negative affect, CoA, and motivation) simultaneously (Table 3, Model 3). This most comprehensive model explores the possibility of all variables being related predictors of N-back performance. The results from this analysis showed that the interactions CoA $\times$ DailyVar and motivation $\times$ DailyVar remain significant predictors of performance, $F(1$, $8,178)=5.06, p<.05$, and $F(1,8,178)=21.87, p<.05$, respectively. The interaction negative affect $\times$ DailyVar again did not explain additional variance in performance, $F(1,8,178)=$ $0.03, p<.05$. The amount of within-person variance in accuracy explained by this model is $9.5 \%$. Thus, accuracy of the N-back task covaries with negative affect, $\mathrm{CoA}$, and motivation, and the three predictors are not independent predictors, but share predictive variance, to the extent that when analyzed together, negative affect becomes nonsignificant. Over and above the theoretical expectations that were tested above, this last analysis revealed shared predictive variance of motivation and CoA. This can be inferred from the reduction in the regression coefficients when comparing Model 1 to 3 (see Table 3), and because the additional variance explained by Model 3 in comparison to Model 2 (predictors motivation and negative affect) is only $0.5 \%$, whereas CoA explained $3.5 \%$ when tested as a single predictor (see Table 2).

\section{Additional Follow-Up Analyses}

The preceding analyses focused on concurrent effects only. In contrast, the causal models presented in the introduction may imply that the effects of negative affect on cognitive performance via motivation and attention may unfold across time as regulatory effort increases. The design of this study allows making first steps toward testing a temporal order in which the variables under investigation affect each other, albeit on the relatively coarse timescale of days. We thus explored whether (a) negative affect
(NA), CoA, and motivation (MOT) on day t had an effect on N-back performance $(\mathrm{NB})$ on day $\mathrm{t}+1$; and (b) N-back performance on day $\mathrm{t}$ had an effect on negative affect, CoA, and motivation on day $t+1$. Neither did the self-report variables predict $\mathrm{N}$-back performance on the subsequent day, estimate $_{\mathrm{NA}-\mathrm{NB}}=-.004$, $t(1,7,977)=-0.1, p>.05$; estimate $_{\mathrm{CoA}-\mathrm{NB}}=.03, t(1,8,175)=$ $1.55, p>.05$; estimate $_{\text {MOT-NB }}=0.04, t(1,8,074)=1.56, p>.05$; nor did N-back performance predict the self-report variables the next day, estimate NB-NA $=0.05, t(1,9,899)=0.74, p>.05$; estimate $_{\mathrm{NB}-\mathrm{CoA}}=-0.01, t(1,9,899)=-0.07, p>.05$; estimate $\left._{\mathrm{NB}-\mathrm{MOT}}=0.07, t(1,9,899)=1.44, p>.05\right)$. Thus, these analyses provide no further information on the temporal ordering of the effects reported in the previous sections.

\section{Discussion}

This study investigated whether variability in WM performance from day to day was coupled with variability in negative affect, control of attention, and motivation within individuals. The results support our hypotheses. Days on which performance was worse than on average were days with increased negative affect and reduced control of attention and motivation. Couplings were primarily found between the different predictors and accuracy of performance, not RT. In addition, individuals differed from each other regarding how systematically their performance varied from day to day. As expected, couplings were apparent for individuals with more systematic daily performance variability. When investigating the different predictors of performance simultaneously, this study identified shared predictive variance among them. That is, days with decreased performance and increased negative affect were days with reduced control of attention and motivation.

\section{Negative Affect, Attention, Motivation, and WM}

Most research describes WM as a trait-like cognitive resource, but recently, its variability within individuals was revealed (Sliwinski et al., 2006). In line with these studies and others showing 
variability in different cognitive ability domains (Nesselroade \& Salthouse, 2004; Rabbitt et al., 2001), this study identified systematic variation in WM performance across days. However, the amount of systematic daily variability was not equal across participants. In some individuals, fluctuations in performance across days were not reliable, in others, they were reliable. These interindividual differences were taken into consideration when testing hypotheses about predictors of performance variations. One contribution this study makes is the thorough description of this procedure (for earlier discussions of this issue, see Rabbitt et al., 2001; Schmiedek, Lövdén, \& Lindenberger, 2008; Wittmann, 1988).

All theoretical assumptions regarding correlates of performance variation (in the individuals who varied more systematically) were supported. Performance was less accurate on days with high levels of negative affect and low levels of control of attention and motivation. Motivation explained most variance in WM variability, followed by attention and negative affect. These differences might partly be explained by varying proximity of the self-report variables to the task. We assessed task-related motivation, whereas attention and affect did not specifically refer to the task. In contrast, RT was not related to affect and attention. Its statistically significant association with motivation seems negligible given that only $0.1 \%$ variance in RT fluctuation could be explained by motivation. That is, participants neither slowed down nor sped up significantly or meaningfully on days with varying levels of affect, attention, and motivation. Together, this study showed systematic within-person associations between negative affect, subjective control of attention, motivation, and accuracy of WM performance. These findings are line with previous results from the COGITO Study revealing an association between effort and enjoyment, two indicators of motivation, and WM performance variability (Brose et al., 2010). Over and above, the findings are consistent with theoretical propositions of the mechanisms that link the different psychological domains, and also suggest that ideas about why experiencing intense negative affect may affect cognition formulated in research on depression and in experimental studies can be translated to within-person functioning in daily life. When mood is disturbed, it may engage attentional processes, and regulatory effort may be activated, both consuming resources not available for task performance (Ellis \& Ashbrook, 1988). In addition, individuals who experience an adverse mood may have a lack of motivation to allocate resources to a task (Hertel, 2000), potentially because they are more motivated to engage in activities which repair mood. Additional research is required to test this latter speculation. Because attentional control as well as motivation are essential for successful performance on tasks requiring cognitive control (Engle, 2002) and because control of attention and motivation may vary for reasons other than impaired mood, for example, because attention is directed at stressful experiences (Sliwinski et al., 2006), these two variables should predict performance independent of their role in the affect-WM relation.

\section{Associations Between Different Predictors of WM}

We applied a hierarchical regression analysis to investigate whether the relation between negative affect and WM performance can partly be explained by reduced control of attention and motivation on days with high negative affect. In line with expectations, these analyses revealed shared predictive variance among the different predictors. Moreover, these findings can be interpreted as being in line with causal ideas of how, or by which mechanism, affect impacts performance. However, this investigation is correlational in nature, and does not allow such inferences to be made. Examples of different possibilities of how the variables may relate are (a) a participant was not motivated to come to our laboratory, which increased his or her negative affect that day and reduced task engagement; and (b) a participant faced some difficulties on a particular day, which resulted in attention being caught up by the problem, in mood being impaired, and in task relevance including the motivation to engage in it being reduced. To complicate matters even further, none of the models may be generally correct or wrong, but the way the causal relationships operate may vary across individuals and across days within individuals. Thus, causal interpretations are not possible based on the present results.

Over and above the hypotheses that were tested, we also explored the joined influence of all three predictors on performance. This revealed that control of attention and motivation also share predictive variance. Thus, the two different paths between affect and performance seem to not be clearly distinguishable. One explanation for this finding may be that subjective control of attention includes a volitional component, that is, the two constructs overlap to some degree. Put differently, whether attention is focused or not may partly depend on whether one wants it to be focused. The way control of attention was operationalized in this study may have contributed to this possibility. Another explanation for why all three predictors are related is that a common cause (e.g., some stressful experience) may underlie increased negative affect and reduced motivation and attention and thus be responsible for the shared predictive variance.

Additional analyses were carried out to explore lead and lagged effects among cognitive performance and negative affect, attentional control, and motivation. Such analyses provide initial evidence for the temporal ordering of the variables. There were no effects of negative affect, attentional control, and motivation (occasion $t$ ) on performance (occasion $t+1$ ), and vice versa. It may well be that the average time delay between occasions (1.5 days) was too long to detect lead and lag associations that are operating within and across days. Future studies would benefit from making more explicit assumptions about the timescales at which negative affect, attentional control, and motivation develop and in which temporal proximity they impact task performance, and vice versa. Data could then be collected in accordance to these ideas and a proposed causal ordering could be tested. Related to this issue, negative affect was assessed as momentary affect in this study, although we assume that our measure in part reflects mood across the day (findings from the COGITO Study on associations between affect and daily stressors are supportive of this view; Brose, Schmiedek, Lövdén, \& Lindenberger, 2011). Collecting repeated information would help in attaining more precise information on the duration of affective states within days. In combination, this additional evidence would help to delineate the extent to which momentary affective disturbances and extended mood characteristics of a day are relevant for performance. 


\section{Future Directions}

A number of questions emerge from this study. The first is why there are effects on performance accuracy but not on RTs. Future studies could address this issue by applying formal modeling such as diffusion modeling of performance (e.g., Ratcliff \& Rouder, 1998; Schmiedek et al., 2009). Diffusion models have been used to investigate processes underlying decision making in simple choice-reaction tasks. The general idea of the diffusion model is that processing as observed with speed and accuracy measures is separated into various underlying components. An analysis of potential variability of these components may reveal why only accuracy was systematically coupled with the different predictors while RT remained unaffected.

A second question is to which extent the findings may hold across different contexts. Is it reasonable to assume that our findings can be replicated in performance situations that may be more relevant to people? For example, is performance at university or at work coupled with daily variability in affect? On the one hand, the proposed links between WM and affective experiences (e.g., attention is directed at affect rather than task performance, motivation is directed at mood repair) should be stable across situations. On the other hand, it seems possible that performance in a laboratory study is more susceptible to minor fluctuations in psychological states than performance outcomes in more meaningful situations. Once the outcome of a task is more relevant, top-down control processes may be activated when experiencing suboptimal states, potentially protecting performance outcomes. Thus, it seems that situational variations and the significance of situations may moderate performance-affect associations.

Third, it is possible that stable dispositions other than the amount of reliable daily variability in WM performance moderate the couplings that were investigated in this study. For example, the impact of daily negative affect may be stronger in individuals that are prone to high levels of negative affect. In these individuals, automatic thought processes that are assumed to be partly responsible for sustained negative affect, such as rumination (NolenHoeksema, Morrow, \& Fredrickson, 1993), may be triggered faster-and rumination about negative affect is among the types of thoughts discussed as detrimental when performing on cognitive tasks (Joormann, 2008). In contrast, individuals high in the ability to control attention may use this ability to buffer the impact of states on performance (see Hofmann, Gschwendner, Friese, Wiers, \& Schmitt, 2008, for an example of how cognitive abilities make a difference when pursuing self-regulatory goals). Future studies should investigate these possibilities by including cognitive measures of executive functioning as moderators of, for example, affect-performance associations.

Fourth, our findings are in line with the proposition that motivation and attention may be triggered by above-average negative affect because the latter indicates a deviation from ideal affective states. Future studies may take into consideration that there are interindividual and situational differences in what ideal affect is (Tamir, 2009; Tsai, 2007). For example, there are situations in which people are oriented toward contra-hedonic states (Riediger, Schmiedek, Wagner, \& Lindenberger, 2009; Tamir, 2009). Such efforts can also be cognitively costly (Riediger et al., 2011). Moreover, individuals differ regarding whether they consider high or low arousal affective states as ideal (Tsai, 2007). For some individuals even high arousal positive states may be subject to regulation effort that is cognitively costly. Related to this issue, the results of this study raise the more general question how positive affect and WM performance are related from day to day. On the one hand, positive affect that is moderately high in arousal may result in increased WM performance, as it is associated with high approach motivation and potentially indicative of an optimal state for task performance (Gable \& Harmon-Jones, 2010; Yerkes \& Dodson, 1908). On the other hand, there is evidence that positive mood impairs executive functioning, potentially because of increased distractibility (Phillips, Bull, Adams, \& Fraser, 2002).

Finally, future studies may want to turn to the individual as unit of analysis when investigating intraindividual couplings between affect and WM by means of dynamic factor analysis, for example. Advantages of such an approach are the possibilities to (a) directly observe interindividual differences in the strength of the couplings that currently can only be speculated about, (b) potentially reveal interindividual differences in the mechanisms that link the different domains, and (c) model changes that may occur in associations within individuals across time (nonstationarity of processes; Molenaar, 2004; Nesselroade, 1991). The advantage of such an approach would be to better understand psychological processes of single individuals - with the added promise to derive formerly hidden nomothetical laws of intraindividual variability and change (e.g., Nesselroade \& Molenaar, 1999).

\section{Conclusion}

Relationships between cognition and emotion, or cognition and motivation have often caught researchers' attention. This study differed from past endeavors by asking whether subtle, daily variations in subjectively accessible states were coupled with performance variability within healthy individuals, assuming that mechanisms relating the different domains at the between-person level should also operate at the within-person level. Results are in favor of this assumption. This study demonstrated that WM performance is not merely a stable attribute in individuals, but that it varies more or less systematically across days. Some of this variability was related to daily fluctuations in affect, attention, and motivation. Future studies should more closely examine the temporal order in which the different variables affect each other and thus provide clear evidence for the theoretical notion supported by our findings - that negative affect reduces cognitive performance by causing a reduction in attention and motivation.

\section{References}

Baddeley, A. D. (1986). Working memory. New York: Oxford University Press.

Beck, A. T. (1967). Depression: Clinical, experimental, and theoretical aspects. New York: Harper \& Row.

Beevers, C. G. (2005). Cognitive vulnerability to depression: A dual process model. Clinical Psychology Review, 25, 975-1002. doi:10.1016/ j.cpr.2005.03.003

Bolger, N., DeLongis, A., Kessler, R. C., \& Schilling, E. A. (1989). Effects of daily stress on negative mood. Journal of Personality and Social Psychology, 57, 808-818.

Brose, A., Schmiedek, F., Lövdén, M., \& Lindenberger, U. (2011). Normal aging dampens the link between intrusive thoughts and negative affect in reaction to daily stressors. Psychology and Aging, 26, 488-502. 
Brose, A., Schmiedek, F., Lövdén, M., Molenaar, P. C. M., \& Lindenberger, U. (2010). Adult age differences in covariation of motivation and working memory performance: Contrasting between-person and withinperson findings. Research in Human Development, 7, 61-78. doi: $10.1080 / 15427600903578177$

Burt, D. B., Zembar, M. J., \& Niederehe, G. (1995). Depression and Memory Impairment - a Metaanalysis of the Association, Its Pattern, and Specificity. Psychological Bulletin, 117, 285-305.

Carver, C. S., \& Scheier, M. F. (1999). Themes and issues in the selfregulation of behavior. In R. S. Wyer, Jr. (Ed.), Perspectives on behavioral self-regulation: Advances in social cognition, (Vol. XII, pp. 1-105). Mahwah, NJ: Erlbaum.

Eccles, J. S., \& Wigfield, A. (2002). Motivational beliefs, values, and goals. Annual Review of Psychology, 53, 109-132.

Ellis, H. C., \& Ashbrook, P. W. (1988). Resource allocation model of the effects of depressed mood states on memory. In K. Fiedler \& J. Forgas (Eds.), Affect, cognition, and social behavior (pp. 25-43). Göttingen: Hogrefe.

Ellis, H. C., Moore, B. A., Varner, L. J., Ottaway, S. A., \& Becker, A. S. (1997). Depressed mood, task organization, cognitive interference, and memory: Irrelevant thoughts predict recall performance. Journal of Social Behavior and Personality, 12, 453-470.

Engle, R. W. (2002). Working memory capacity as executive attention. Current Directions in Psychological Science, 11, 19-23.

Gable, P. A., \& Harmon-Jones, E. (2010). The motivational dimensional model of affect: Implications for breadth of attention, memory, and cognitive categorization. Cognition and Emotion, 24, 322-337.

Gendolla, G. H. E. (2000). On the impact of mood on behavior: An integrative theory and review. Review of General Psychology, 4, 378408.

Grant, M. M., Thase, M. E., \& Sweeney, J. A. (2001). Cognitive disturbance in outpatient depressed younger adults: Evidence of modest impairment. Biological Psychiatry, 50, 35-43.

Harvey, P. O. (2004). Executive functions and updating of the contents of working memory in unipolar depression. Journal of Psychiatric Research, 38, 567-576. doi:10.1016/j.jpsychires.2004.03.003

Hasher, L., Zacks, R. T., \& May, C. P. (1999). Inhibitory control, circadian arousal, and age. In D. Gopher \& A. Koriat (Eds.), Attention and performance XVII: Cognitive regulation of performance: Interaction of theory and application (pp. 653-675). Cambridge, MA: The MIT Press.

Hautzinger, M. (1988). Die CES-D Skala. Ein Depressionsmeßinstrument für Untersuchungen in der Allgemeinbevölkerung. [The CES-D scale: A measure of depressive symptoms for studying normal populations] $\mathrm{Di}$ agnostica, 34, 167-173.

Hautzinger, M., \& Bailer, M. (1993). ADS-Allgemeine Depressionsskala. Weinheim: Beltz.

Hertel, P. T. (2000). The cognitive-initiative account of depression-related impairments in memory. Psychology of Learning and Motivation: Advances in Research and Theory, 39, 47-71.

Hertel, P. T., \& Hardin, T. S. (1990). Remembering with and without awareness in a depressed mood-Evidence of deficits in initiative. Journal of Experimental Psychology-General, 119, 45-59.

Hertel, P. T., \& Rude, S. S. (1991). Depressive deficits in memory-Focusing attention improves subsequent recall. Journal of Experimental Psychology-General, 120, 301-309.

Hofmann, W., Gschwendner, T., Friese, M., Wiers, R. W., \& Schmitt, M. (2008). Working memory capacity and self-regulatory behavior: Toward an individual differences perspective on behavior determination by automatic versus controlled processes. Journal of Personality and Social Psychology, 95, 962-977. doi:10.1037/A0012705

Joormann, J. (2008). Cognitive aspects of depression. In I. H. Gotlib \& C. Hammen (Eds.), Handbook of depression (pp. 298-321). New York: Guilford Press.

Kane, M. J., Brown, L. H., Little, J. C., \& Silvia, P. J. (2007). An experience-sampling study of working memory and executive control of daily life. Psychological Science, 18, 614-621.

Kane, M. J., Conway, A. R. A., Hambrick, D. Z., \& Engle, R. W. (2007). Variation in working memory capacity as variation in executive attention and control. In A. R. A. Conway, C. Jarrold, M. J. Kane, A. Miyake \& J. N. Towse (Eds.), Variation in working memory (pp. 21-48). New York: Oxford University Press.

Kuhl, J. (1987). Action control: The maintenance of motivational states. In F. Halisch \& J. Kuhl (Eds.), Motivation, intention, and volition (pp 279-307). Berlin: Springer.

Kuhl, J., \& Helle, P. (1986). Motivational and Volitional Determinants of Depression - the Degenerated-Intention Hypothesis. Journal of Abnormal Psychology, 95, 247-251.

Larsen, R. J. (2000). Toward a science of mood regulation. Psychological Inquiry, 11, 129-141

Li, S.-C., Huxhold, O., \& Schmiedek, F. (2004). Aging and attenuated processing robustness. Evidence from cognitive and sensorimotor functioning. Gerontology, 50, 28-34.

Lindenberger, U., \& Potter, U. (1998). The complex nature of unique and shared effects in hierarchical linear regression: Implications for developmental psychology. Psychological Methods, 3, 218-230.

Lövdén, M., Li, S. C., Shing, Y. L., \& Lindenberger, U. (2007). Withinperson trial-to-trial variability precedes and predicts cognitive decline in old and very old age: Longitudinal data from the Berlin Aging Study. Neuropsychologia, 45, 2827-2838.

Lyubomirsky, S., Tucker, K. L., Caldwell, N. D., \& Berg, K. (1999). Why ruminators are poor problem solvers: Clues from the phenomenology of dysphoric rumination. Journal of Personality and Social Psychology, 77, 1041-1060

Marsh, H. W., Byrne, B. M., \& Shavelson, R. (1988). A multifaceted academic self-concept: Its hierarchical structure and its relation to academic achievement. Journal of Educational Psychology, 80, 366-380.

Mischel, W., \& Shoda, Y. (1995). A cognitive-affective system theory of personality: Reconceptualizing situations, dispositions, dynamics, and invariance in personality structure. Psychological Review, 102, $246-$ 268.

Molenaar, P. C. M. (2004). A manifesto on psychology as idiographic science: Bringing the person back into scientific psychology, this time forever. Measurement, 2, 201-218

Nesselroade, J. R. (1991). The warp and the woof of the developmental fabric. In R. Downs, L. Liben, \& D. S. Palermo (Eds.), Visions of aesthetics, the environment, \& development: The legacy of Joachim F. Wohlwill (pp. 213-240). Hillsdale, NJ: Erlbaum.

Nesselroade, J. R., \& Molenaar, P. C. M. (1999). Pooling lagged covariance structures based on short, multivariate time series for dynamic factor analysis. In R. H. Hoyle (Ed.), Statistical strategies for small sample research (pp. 223-250). Thousand Oaks, CA: Sage.

Nesselroade, J. R., \& Salthouse, T. A. (2004). Methodological and theoretical implications of intraindividual variability in perceptual-motor performance. Journal of Gerontology: Psychological Sciences, 59B P49-P55

Nolen-Hoeksema, S., Morrow, J., \& Fredrickson, B. (1993). Response styles and the duration of episodes of depressed mood. Journal of Abnormal Psychology, 102, 20-28.

Nolen-Hoeksema, S., Wisco, B. E., \& Lyubomirsky, S. (2008). Rethinking rumination. Perspectives on Psychological Science, 3, 400-424.

Oberauer, K. (2005). Binding and inhibition in working memory: Individual and age differences in short-term recognition. Journal of Experimental Psychology: General, 3, 368-387.

Phillips, L. H., Bull, R., Adams, E., \& Fraser, L. (2002). Positive mood and executive function: Evidence from Stroop and fluency tasks. Emotion, 2 12-22.

Rabbitt, P., Osman, P., Moore, B., \& Stollery, B. (2001). There are stable individual differences in performance variability, both from moment to 
moment and from day to day. The Quarterly Journal of Experimental Psychology, 54A, 981-1003.

Ratcliff, R., \& Rouder, J. N. (1998). Modeling response times for two choice-decisions. Psychological Science, 9, 347-356.

Richards, J. M., \& Gross, J. J. (2000). Emotion regulation and memory: The cognitive costs of keeping one's cool. Journal of Personality and Social Psychology, 79, 410-424.

Riediger, M., Schmiedek, F., Wagner, G. G., \& Lindenberger, U. (2009). Seeking pleasure and seeking pain: Age-related differences in pro- and contra-hedonic motivation from adolescence to old age. Psychological Science, 20, 1529-1535.

Riediger, M., Wrzus, C., Schmiedek, F., Wagner, G. G., \& Lindenberger, U. (2011). Is seeking bad mood cognitively demanding? Contra-hedonic orientation and working-memory capacity in everyday life. Emotion, 11, 656-665. doi:10.1037/a0022756

Röcke, C. (2006). Intraindividual variability in positive and negative affect: Age-related and individual differences in magnitude and coupling with cognitive performance. (Doctoral dissertation, Free University Berlin, Germany). Retrieved from http://www.diss.fu-berlin.de/2006/669/

Rogers, M. A., Kasai, K., Koji, M., Fukuda, R., Iwanami, A., Nakagome, K., ... Kato, N. (2004). Executive and prefrontal dysfunction in unipolar depression: A review of neuropsychological and imaging evidence. Neuroscience Research, 50, 1-11. doi:10.1016/j.neures.2004 .05 .003

Ruppert, D., Wand, M. P., \& Carroll, R. J. (2003). Semiparametric regression. Cambridge: Cambridge University Press.

Ryan, R. M., \& Deci, E. L. (2000). Self-determination theory and the facilitation of intrinsic motivation, social development, and well-being. American Psychologist, 55, 68-78.

Salthouse, T. A., \& Berish, D. E. (2005). Correlates of within-person (across-occasion) variability in reaction time. Neuropsychology, 19, 77-87.

Sarason, I. G., Sarason, B. R., Keefe, D. E., Hayes, B. E., \& Shearin, E. N. (1986). Cognitive interference: Situational determinants and traitlike characteristics. Journal of Personality and Social Psychology, 51, 215226.

Scheibe, S., \& Blanchard-Fields, F. (2009). Effects of regulating emotions on cognitive performance: What is costly for young adults is not so costly for older adults. Psychology and Aging, 24, 217-223. doi: 10.1037/A0013807

Schmiedek, F., Bauer, C., Lövdén, M., Brose, A., \& Lindenberger, U. (2010). Cognitive enrichment in old age: Web-based training programs. Gerontology, 23, 59-67.

Schmiedek, F., Lövdén, M., \& Lindenberger, U. (2008). The COGITO Study: Investigating the Ergodicity Assumption in younger and older age groups. Paper presented at the Cognitive Aging Conference, Atlanta.

Schmiedek, F., Lövdén, M., \& Lindenberger, U. (2009). On the relation of mean reaction time and intraindividual reaction time variability. Psychology and Aging, 24, 841-857.

Schmiedek, F., Lövdén, M., \& Lindenberger, U. (2010). Hundred days of cognitive training enhance broad cognitive abilities in adulthood: Findings from the COGITO study. Frontiers in Aging Neuroscience, 2, 1-10. doi:10.3389/fnagi.2010.00027

Schwarzer, R., Diehl, M., \& Schmitz, G. S. (1999). Self-regulation. Retrieved from http://userpage.fu-berlin.de/ health/selfreg_e.htm

Seibert, P. S., \& Ellis, H. C. (1991). Irrelevant thoughts, emotional mood states, and cognitive task performance. Memory \& Cognition, 19, 507513

Shamosh, N. A., \& Gray, J. R. (2007). The relation between fluid intelligence and self-regulatory depletion. Cognition and Emotion, 8, 18331843.

Siegle, G. J., Ingram, R. E., \& Matt, G. E. (2002). Affective interference: An explanation for negative attention biases in dysphoria? Cognitive Therapy and Research, 26, 73-87. doi:0147-5916/02/0200-0073/0

Siegler, R. S. (1994). Cognitive variability: A key to understanding cognitive development. Current Directions in Psychological Science, 3 , $1-5$.

Singer, J. D., \& Willett, J. B. (2003). Applied longitudinal data analysis: Modeling change and event occurrence. New York: Oxford University Press.

Sliwinski, M. J., Smyth, J. M., Hofer, S. M., \& Stawski, R. S. (2006). Intraindividual coupling of daily stress and cognition. Psychology and Aging, 21, 545-557.

Tamir, M. (2009). What do people want to feel and why? Pleasure and utility in emotion regulation. Current Directions in Psychological Science, 18, 101-105. doi:10.1111/j.1467-8721.2009.01617.x

Tsai, J. L. (2007). Ideal affect: Cultural causes and behavioral consequences. Perspectives on Psychological Science, 2, 242-259.

Watson, D., Clark, L. A., \& Tellegen, A. (1988). Development and validation of brief measures of positive and negative affect: The PANAS scales. Journal of Personality and Social Psychology, 54, 1063-1070.

Wittmann, W. W. (1988). Multivariate reliability theory. Principles of symmetry and successful validation strategies. In J. R. Nesselroade \& R. B. Cattell (Eds.), Handbook of multivariate experimental psychology (pp. 505-556). New York: Plenum Press.

Yeo, G. B., \& Neal, A. (2004). A multilevel analysis of effort, practice, and performance: Effects of ability, conscientiousness, and goal orientation. Journal of Applied Psychology, 89, 231-247.

Yerkes, R. M., \& Dodson, J. D. (2008). The relation of strength of stimulus to rapidity of habit-formation. Journal of Comparative Neurology and Psychology, 18, 459-482.

Received October 17, 2010

Revision received May 9, 2011

Accepted May 11, 2011 